\title{
Inferior Vena Cava Filters as a Potential Non-immune Cause of Low Serum Haptoglobin
}

Laura Cooling ${ }^{*}$, Donald Giacherio and Matthew Elkins

Departments of Pathology, University of Michigan, Ann Arbor MI and SUNY Upstate Medical University, Syracuse, New York, USA

*Corresponding Author: Laura Cooling, MD, MS, Associate Professor, Pathology Associate Director, Transfusion Medicine University of Michigan Hospitals 2F225 UH Blood Bank, Box 00541500 East Medical Center Drive Ann Arbor, MI 48109-0054, USA ,Tel: (734) 936-0695; E-mail: 1cooling@med.umich.edu

Received Date: March 28, 2017; Accepted date: April 29, 2017; Published date: May 05, 2017

Copyright: $\odot 2017$ Cooling L, et al. This is an open-access article distributed under the terms of the Creative Commons Attribution License, which permits unrestricted use, distribution, and reproduction in any medium, provided the original author and source are credited.

\begin{abstract}
Objective: Serum haptoglobin level is commonly used as a marker of hemolysis. We hypothesized that inferior vena cava (IVC) filters may be a potential non-immune etiology for subclinical hemolysis and low haptoglobin in some patients, after encountering a patient with an IVC filter, mild macrocytic anemia and unexplained severe haptoglobinemia. IVC filters can be associated with pressure gradients and turbulent blood flow in the presence of trapped clots and thrombus formation.
\end{abstract}

Methods: Prospective study in patients undergoing IVC filter placement at our institution over a 3-month period in 2008. All patients had a haptoglobin level prior to filter placement, and then daily for a period of 3-14 days. The absolute and relative change in haptoglobin compared to pre-procedure levels was determined. Results were evaluated by t-test (paired, 2-tail). Statistics and graphics were performed with commercial software.

Results: A total of 22 patients underwent IVC filter placement. Four patients were excluded due to low preprocedure haptoglobin levels. At least one post-procedure haptoglobin values was available until day 3 in $18 / 18$ eligible patients. Nine patients were followed for a period of 1-2 weeks. Although the majority of patients had an increase in haptoglobin after IVC filter placement, $3 / 9(30 \%)$ had $>50 \%$ decrease in haptoglobin by day 7 , including one patient with severe haptoglobinemia by day 14. Patients with decreased haptoglobin levels also had decreases in platelet count over the same period.

Conclusion: These results suggest that IVC filters may be another non-immune, device-related cause of decreased haptoglobin in some patients.

Keywords: Haptoglobin; Inferior vena cava filter; Pulmonary embolism; Deep venous thrombosis

Abbreviations: DVT: Deep Venous Thrombosis; IVC: Inferior Vena Cava; MCV: Mean Corpuscular Volume; PE: Pulmonary Embolism; PT: Prothrombin Time; PTT: Partial Thromboplastin Time; RBC: Red Blood Cell Unit; RDW: Red Cell Distribution Width

\section{Introduction}

Haptoglobin is an a2-glycoprotein that acts as a hemoglobin scavenger, binding free hemoglobin dimers and facilitating their clearance via CD163 on macrophages [1,2]. Haptoglobin levels are exquisitely sensitive to even mild hemolysis, due to rapid clearance of haptoglobin-hemoglobin complexes (minutes) and limited hemoglobin-binding capacity [1]. Specifically, the ratio of haptoglobin: hemoglobin in $1 \mathrm{~mL}$ hemolyzed blood is 1:1000 [3], which suggests that hemolysis of $15-20 \mathrm{ml}$ of blood would be sufficient to deplete haptoglobin in an average adult total blood volume. Circulating haptoglobin is primarily synthesized by the liver; however, localized haptoglobin synthesis has been identified in the brain, skin, lung, kidney, spleen, thymus, and heart $[1,4]$.

Haptoglobin levels are decreased in transfusion reactions, acute and chronic immune hemolytic anemias, sickle cell disease and other congenital anemias [1,5]. Haptoglobin is also decreased in the setting of mechanical and shear-induced hemolysis including severe aortic stenosis, heart valves, thrombectomy, extracorporeal membrane oxygenation, and cardiac bypass surgery [6-11]. In some instances, non-immune, mechanical hemolysis has been initially confused for a hemolytic transfusion reaction [7]. Clinically, haptoglobin levels $<25$ $\mathrm{mg} / \mathrm{dL}$ are highly sensitive $(83-92 \%)$ and specific (96-98\%) for intravascular hemolysis [1].

Previously, we encountered a neurology patient with mild pancytopenia, who also had laboratory evidence of chronic low grade hemolysis as evidenced by a persistently low haptoglobin $(1 \mathrm{mg} / \mathrm{dl})$, macrocytic anemia and polychromasia. After an extensive workup, his pancytopenia was attributed to marrow suppression by anti-seizure medications, however, an explanation for his low haptoglobin was not identified. A review of the patient's history revealed a history of deep venous thrombosis (DVT) and pulmonary embolus (PE), necessitating the placement of an inferior vena cava (IVC) filter. We hypothesized that the patient may have had low grade hemolysis secondary to the IVC filter. A review of the literature found no studies examining the impact of IVC filters on haptoglobin levels. We therefore performed a small prospective study of serial haptoglobin levels in patients undergoing IVC filter placement at our institution to determine whether IVC filters might be a non-immune etiology for low haptoglobin in some patients. 
Page 2 of 8

\section{Materials and Methods}

\section{Study design}

The study was a prospective, longitudinal analysis of serum haptoglobin levels in all adult patients who underwent placement of an IVC filter at the University of Michigan between August and November, 2008. There were no exclusion criteria regarding patient underlying diagnosis, anticoagulation or other co-morbidities.

A serum haptoglobin level was added to routine pre-operative chemistries in all patients immediately prior to IVC placement (day 0). In patients requiring routine serum chemistries post-operatively, a haptoglobin was added off-line for a period of 3 to 5 days postoperatively, as available. In some patients, samples were available for testing for 7-14 days post-procedure. The study was approved by the institutional medical investigation review board.

Patient demographics included age, sex, reason for IVC filter placement and anticoagulation. In addition, a chart review was performed for confounding variables within the observational period including thrombectomy, thrombolytic therapy, prosthetic valves, tricuspid valve regurgitation and pulmonary hypertension, blood transfusion, and infection [5-14]. In select patients, a review of related laboratory values during the study period was performed. Laboratory studies included total bilirubin, lactate dehydrogenase ( $\mathrm{LDH}$, range 120-240 IU/L), hemoglobin (Hgb, range 12-16 gm/dl), mean corpuscular volume (MCV, range 80-100 fl), red cell distribution (RDW, range 11.5-15.5\%), prothrombin time (PT, range, $9.5-11.7 \mathrm{~s}$ ), partial thromboplastin time (PTT, range 23-32 s), fibrinogen (range, $150-450 \mathrm{mg} / \mathrm{dL}$ ) and platelet count (range 150-450 K/ $\mu \mathrm{l}$ ). In transfused patients, the results of the red cell antibody screen on admission, prior to IVC filter placement and following transfusion (1 to 6 months posttransfusion) were reviewed to identify any new RBC alloantibodies.

\section{Haptoglobin measurement}

Haptoglobin was measured by an immunoturbidimetric assay on a Roche COBAS Integra 800 automated chemistry analyzer (Roche Diagnostics Corporation, Indianapolis, IN). In the assay, monoclonal anti-human haptoglobin reacts with haptoglobin in the sample, with the formation of immune complexes and an increase in sample turbidity at $340 \mathrm{~nm}$. Results were reported as $\mathrm{gm} / \mathrm{dL}$. The normal range during the study period was $22-239 \mathrm{mg} / \mathrm{dl}$. Haptoglobin phenotyping (Hp1, Hp2) was not performed [2,15].

\section{Statistical analysis}

Haptoglobin results were examined in each patient relative to their day 0 or pre-procedure serum sample. Patients with depleted, low haptoglobin levels upon study entry (day 0) were excluded from analysis. For each patient, the absolute difference and the percent (\%) change in haptoglobin relative to day 0 was determined. Results were compared by standard and paired t-test. Graphics and commercial software were performed with commercial software (Kaleidograph, Synergy Software, Reading, PA).

\section{Results}

\section{Patients}

A total of 22 patients underwent IVC placement during the 3month study period. Four patients were excluded during analysis due to extremely low day 0 haptoglobin levels (haptoglobin=1-11 gm/dl). In three patients, a predisposing condition or treatment known to decrease haptoglobin was identified: end-stage liver disease, thrombectomy and/or thrombolytic therapy $[1,7,8]$. For the remaining 18 patients, the mean haptoglobin on day 0 was $274 \pm 142 \mathrm{mg} / \mathrm{dl}$ (median 283, range 59-551). There was no correlation between patient age and baseline haptoglobin levels (data not shown, $\mathrm{R}=0.004$ ) [15].

Patient demographics for eligible patients are shown in Table 1. The median age was 58 years, with a nearly equal distribution of men and women. The majority of patients had a recent history or were currently receiving anticoagulation, including two patients recently on warfarin [16]. Almost $60 \%$ of IVC filters were placed in patients with an acute thrombosis, including two patients with both DVT and PE. Most patients had a Gunther-Tulip retrievable IVC filter except two patients, who received a Greenfield filter. Five patients were discharged by postoperative day 3 and were not available for further studies.

\begin{tabular}{|c|c|}
\hline Variable & No. patients \\
\hline No. Patients & 18 \\
\hline Median age, years (range) & $58(20-88)$ \\
\hline $\operatorname{Sex}(M / F)$ & $8 / 10$ \\
\hline Primary diagnosis (\%) & - \\
\hline Cancer & $6(33 \%)$ \\
\hline Surgery & $5(28 \%)$ \\
\hline Cerebral hemorrhage & $4(22 \%)$ \\
\hline Other & $3(17 \%)$ \\
\hline Previous anticoagulation & $13(72 \%)$ \\
\hline Systemic anticoagulation & $9(50 \%)$ \\
\hline Anti-platelet & $4(22 \%)$ \\
\hline Prophylactic IVC placement & $8(44 \%)$ \\
\hline Surgery & 3 \\
\hline Active/recent bleeding & 5 \\
\hline Active thrombosis & $10(56 \%)$ \\
\hline DVT & 8 \\
\hline PE & 4 \\
\hline
\end{tabular}

Table 1: Patient demographics.

\section{Impact of IVC placement on haptoglobin levels}

There was no significant change in haptoglobin levels on day 1 (mean $240 \pm 115 \mathrm{mg} / \mathrm{dl}, \mathrm{p}=0.86$ paired t-test). By day 3, the mean haptoglobin level was actually higher than day $0(395 \pm 139, \mathrm{p}=0.42)$. 
By approximately 1 week, the mean haptoglobin was $89 \%$ of preprocedure values $(213 \pm 89, \mathrm{p}=0.29)$.

An analysis of individual patients revealed two distinct subgroups (Figure 1A). The majority of patients (group 1) exhibited an absolute and relative increase in haptoglobin following IVC filter placement, with peak haptoglobin on day 3 (mean $299 \pm 140, p=0.12$ ). In 10 patients with serial haptoglobins for more than 3 days, the relative change in haptoglobin continued to increase until day 5 (Figure $1 \mathrm{~A}$, $169 \%$ increase over day 0 ). The mean haptoglobin by day 7 in group 1 was $244 \pm 80 \mathrm{mg} / \mathrm{dl}, \mathrm{p}=0.86 ; \mathrm{n}=6)$.

In three patients (group 2), haptoglobin levels declined by over $50 \%$ by day 7 (Figures $1 \mathrm{~A}$ and $\mathrm{B}$ ). In one patient, a haptoglobin was available 2 weeks post-procedure. As shown in Figure 1, haptoglobin levels were $2 \%$ of pre-procedure levels and below the normal range.

\section{Comparison of group 1 and group 2 patients}

We compared group 1 and group 2 patients relative to underlying disease, reasons for IVC filter placement, transfusion history, infection, anticoagulation and liver function (Table 2). Group 1 patients tended to be older and were more likely to receive an IVC filter for PE prophylaxis. There were an equal number of patients in both groups who received an IVC filter following an acute thrombotic event. In both groups, the majority of patients were not placed on systemic anticoagulation following filter placement. One patient (group 2) had a severe PE with tricuspid regurgitation and pulmonary hypertension, which has been associated with lower haptoglobin levels [12,13]. This patient also received thrombolytic therapy. No patient had a diagnosis of end-stage liver disease, valvular heart disease or immune-mediated hemolysis $[1,5,11]$.

One interesting difference was a higher pre-procedure haptoglobin in group 2 (434 \pm 174 versus $240 \pm 115$ group $1, p=0.03$ ). By day 7 , however, the mean haptoglobin had fallen $67 \%$ and was actually $42 \%$ lower than group 1 (Table 2). As an acute phase reactant, haptoglobin levels can increase in the face of surgery, infection and inflammation [14]. There were an equal proportion of patients in both groups with at least one documented infection (admission to day +7 ). In group 1, documented infections included catheter-associated bacteremia, pneumonia, urosepsis, and osteomyelitis. Infections in group 2 were limited to $C$. difficile colitis and a superficial surgical wound infection. Two patients in group 1 had elevated C-reactive protein with normal haptoglobin levels $[5,14]$. Only one patient (group 1) had recently been on warfarin, which is reported to cause mild increases in haptoglobin and other acute phase proteins [16]. No patient was typed for Hp1/Hp2 haptoglobin phenotype, which has been linked to differences in haptoglobin levels $[2,15]$.

We also looked at transfusion history. There were an equal proportion of patients in both groups who were transfused with RBC in the first week after IVC filter placement (day -2 to day $+7 ; 50 \%$ group $1 \mathrm{vs}$. 33\% group 2). In transfused patients, there were no cases of new RBC alloantibodies after 1 to 6 months follow-up.

\section{Laboratory analysis of low haptoglobin levels post-IVC placement}

A chart review was performed for all three patients in group 2 to determine whether there was other laboratory evidence for hemolysis. Laboratories included hemoglobin, platelet count, total bilirubin,
MCV, RDW, and coagulation studies (PT, PTT, fibrinogen). There was no available $\mathrm{LDH}$, reticulocyte or peripheral smear in any patient.

Two patients showed mild decreases in hemoglobin accompanied by rising $\mathrm{MCV}$ and RDW. In addition, all 3 patients displayed a transient $32 \%$ to $42 \%$ decrease in platelet count $3-5$ days post-procedure. There was no clinical evidence of disseminated intravascular coagulopathy. In non-anticoagulated patients $(n=2)$, serial coagulation studies showed no evidence of a consumptive coagulopathy. The total bilirubin in all three patients remained within normal limits.

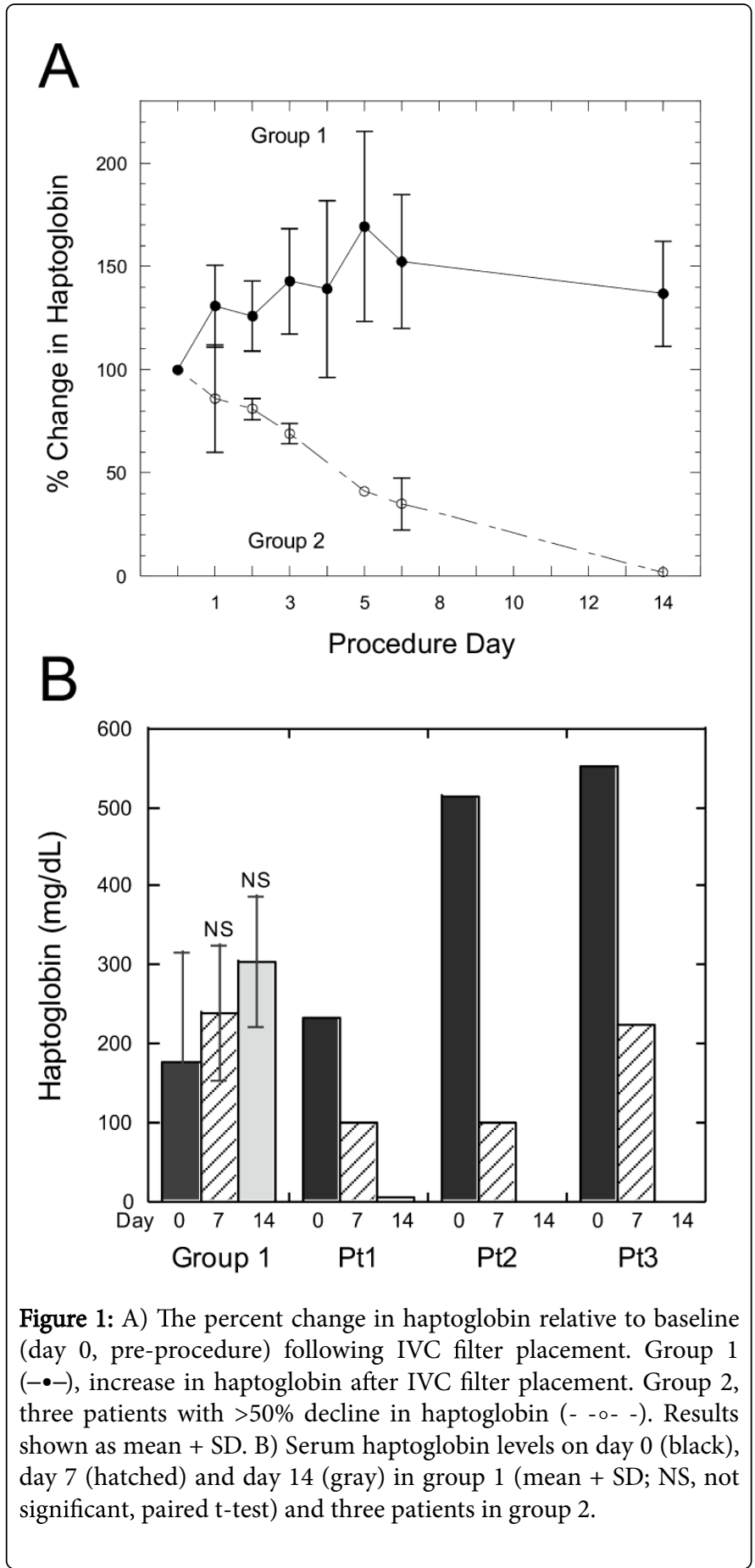


Citation: Cooling L, Giacherio D, Elkins M (2017) Inferior Vena Cava Filters as a Potential Non-immune Cause of Low Serum Haptoglobin. J

Page 4 of 8

The falling hemoglobin and rising MCV and RDW observed in two patients suggested a marrow response to RBC losses. In the absence of reticulocyte counts, we plotted the $\mathrm{MCV}$ against the RDW in all patients from day -3 to day +10 (Figure 2). The MCV and RDW have been used as an initial screening tool in differentiating anemia due to iron deficiency, aplastic anemia, myelodysplastic syndrome and hemolytic anemias [17-20]. Consistent with a reticulocyte response, there was positive linear correlation between the MCV and RDW in hemolytic anemia patients and $2 / 3$ patients in group 2 (Figure $2, \mathrm{~A}-\mathrm{C}$ ). In contrast, group 1 patients showed a normal inverse relationship between MCV and RDW [21], with individual patients showing no change or a negative association (Figure 2, D-I).

\begin{tabular}{|c|c|c|}
\hline & Group 1 & Group 2 \\
\hline No. Patients & 6 & 3 \\
\hline Haptoglobin, mg/dl & - & - \\
\hline Pre-IVC & $240 \pm 115$ & $434 \pm 174$ \\
\hline Day 7 & $244 \pm 80$ & $142 \pm 72$ \\
\hline Patient age, median (range) & $75(20-83)$ & $52(48-58)$ \\
\hline $\operatorname{Sex}(M / F)$ & $2 / 4$ & $2 / 1$ \\
\hline Prior anticoagulation & 3 & 1 \\
\hline Warfarin & 1 & 0 \\
\hline Reason for IVC Filter & - & - \\
\hline Prophylactic & 3 & 0 \\
\hline Acute thrombosis & 3 & 3 \\
\hline DVT & 2 & 3 \\
\hline PE & 1 & 2 \\
\hline Anticoagulant-related bleeding & 3 & 1 \\
\hline Brain hemorrhage or tumor & 1 & 2 \\
\hline Pulmonary hypertension & 0 & $1^{*}$ \\
\hline Tricuspid regurgitation & 0 & $1^{*}$ \\
\hline Thrombolytic Therapy & 0 & $1^{*}$ \\
\hline Anticoagulation post-IVC filter & 2 & 1 \\
\hline Decreased platelets $†$ & 1 & 3 \\
\hline RBC transfusion (day -2 to +7 ) & 3 & 1 \\
\hline No. RBC units transfused & $2(2-4)$ & 4 \\
\hline New RBC alloantibodies $\ddagger$ & 0 & 0 \\
\hline Infection $\pi$ & 4 & 2 \\
\hline \multicolumn{3}{|c|}{$\begin{array}{l}\text { * Patient treated with tissue plasminogen activator for large saddle PE. Patient had acute, transient tricuspid valve regurgitation and pulmonary hyp } \\
\text { associated with the event, which resolved with treatment. } \\
\dagger \text { Greater than } 25 \% \text { decrease in platelets from pre-procedure. Decrease in platelets usually observed in the first 24-48 hours post-IVC placement. } \\
\text { † Development of new RBC alloantibodies following RBC transfusion. } \\
\text { ףInfection: urinary tract infection and bacteremia (1), osteomyelitis (1), C. difficile (1), catheter-associated line infection (1), wound infection (1), pneumonia (1). }\end{array}$} \\
\hline
\end{tabular}

Table 2: Patients with serial haptoglobin measurements for 7 days post-IVC filter placement. 
Citation: Cooling L, Giacherio D, Elkins M (2017) Inferior Vena Cava Filters as a Potential Non-immune Cause of Low Serum Haptoglobin. J
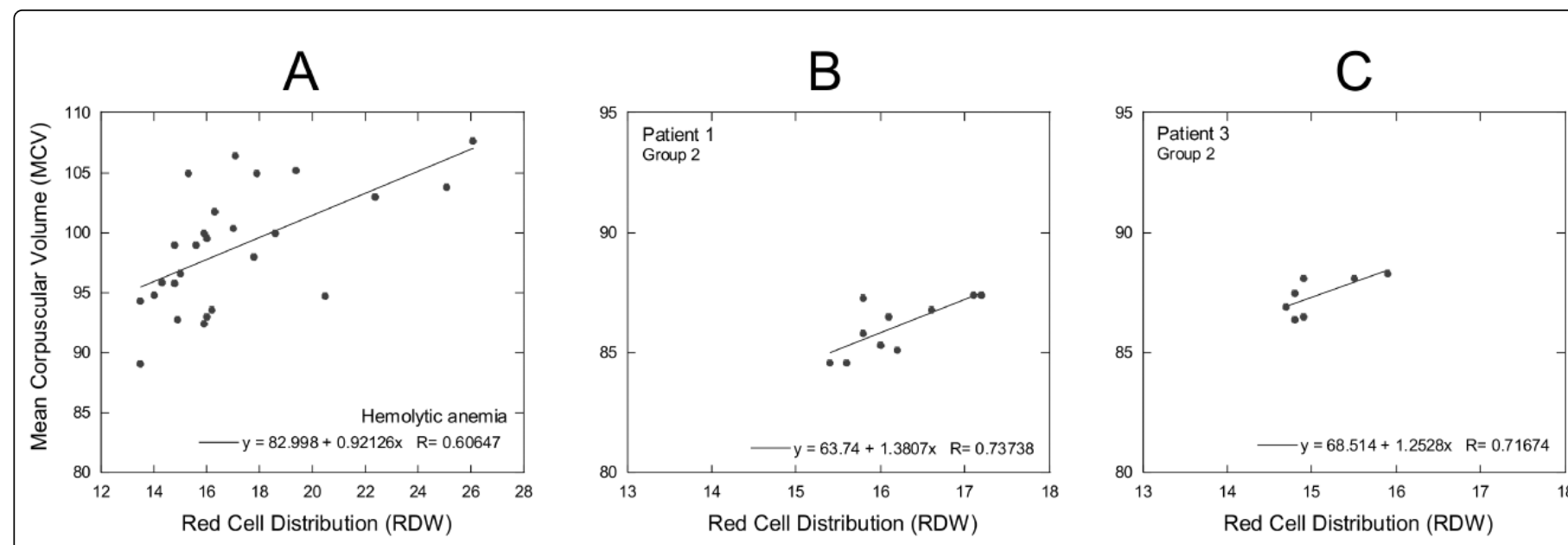

D

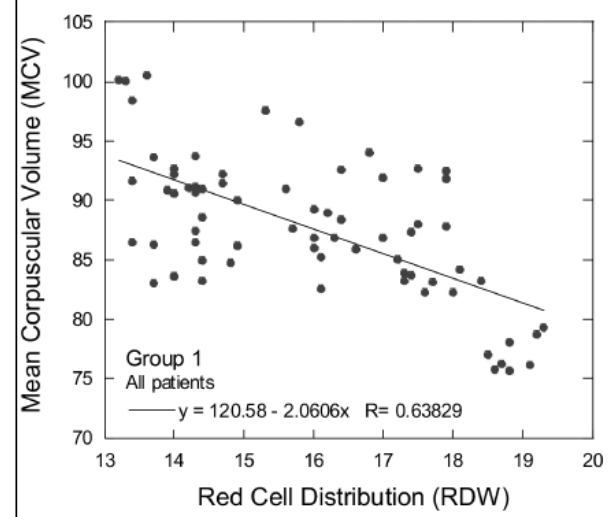

G

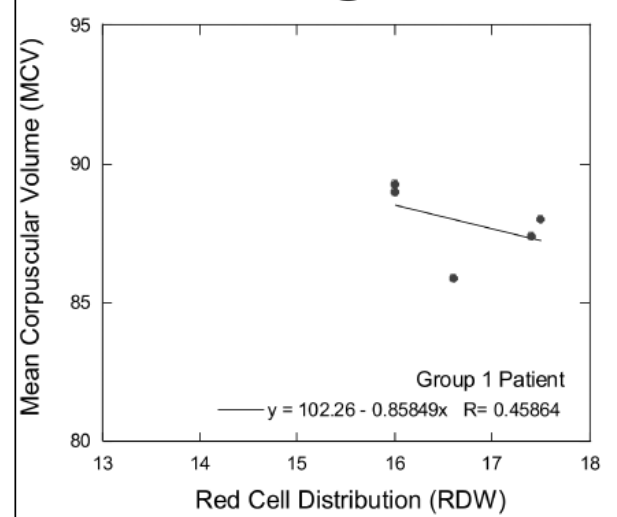

E

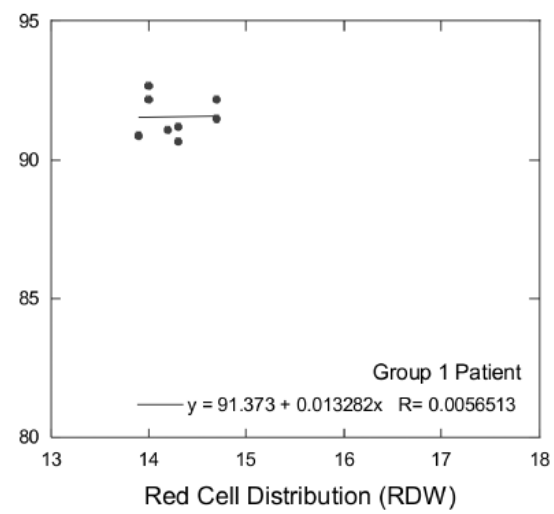

$\mathrm{H}$

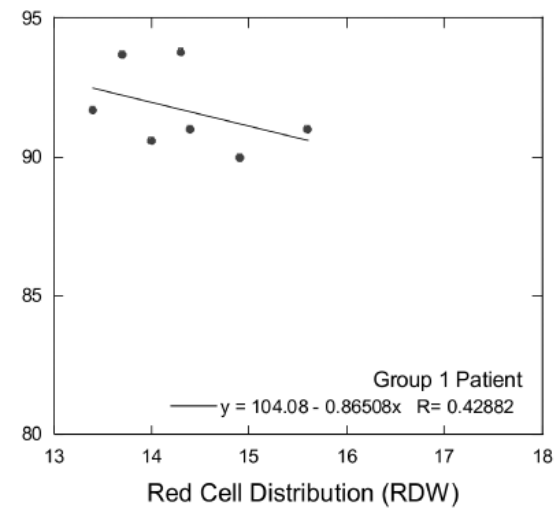

$\mathrm{F}$
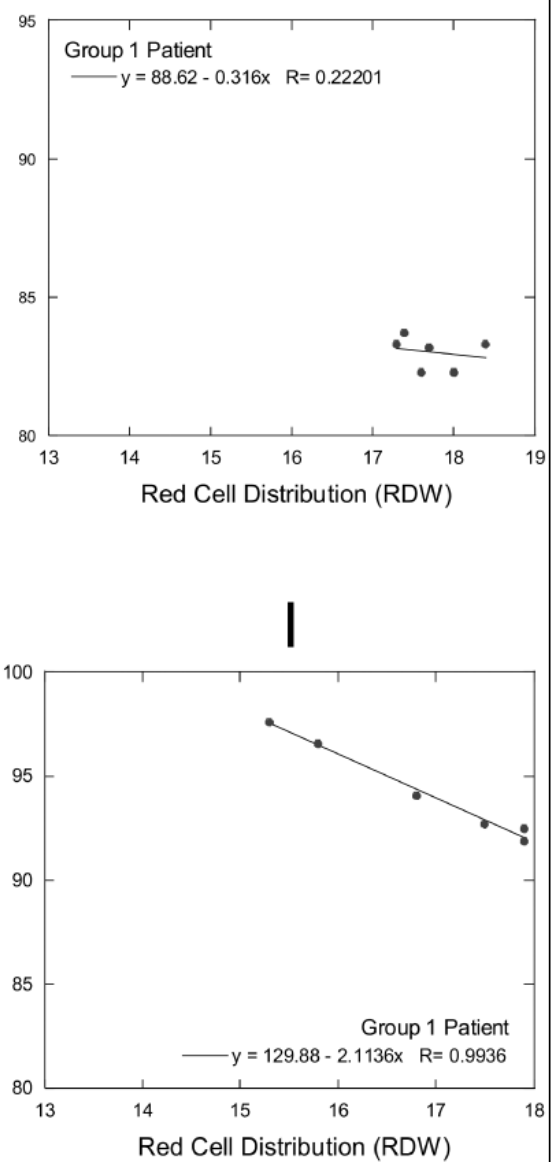

Figure 2: Relationship between MCV and RDW in patients with A) active hemolysis and B, C) group 2 patients. Hemolytic anemia patients included patients due to immune (autoimmune hemolytic anemia) and non-immune hemolysis (thrombotic (hrombocytopenia purpura) with haptoglobin $<10$ and reticulocytosis (3-11\%). Panel D, aggregate data of group 1 patients showing the normal inverse relationship between MCV and RDW [15]. Panels E-I, individual group 1 patients. Data for IVC filter patients ranged from day -3 to day +10 . 


\section{Case histories for patients with decrease haptoglobin post- IVC filter}

The group 2 patients with decreased haptoglobin following IVC filter placement are summarized below:

Case 1: The patient was a 58-year-old, morbidly obese woman with a history of abdominal surgery one month earlier, which was complicated by wound dehiscence, a superficial wound infection and prolonged immobility. She presented to the emergency room with right leg pain, hypotension and dyspnea due to a large saddle PE with multiple emboli to both lungs and a right lower extremity DVT. An echocardiogram showed severe right ventricular dysfunction, moderate tricuspid regurgitation and probable pulmonary hypertension. She underwent emergent IVC filter placement, antithrombolytic therapy with tissue plasminogen activator (2 hours), and heparin anticoagulation. During her first 18 hours, she required transfusion with 4 units of red blood cells (RBC) due to on-going bleeding from a central venous catheter. Bleeding stopped after temporary discontinuation of heparin and re-dressing of the line site. The patient was started on coumadin anticoagulation on postoperative day 4.

Her haptoglobin on admission was $515 \mathrm{mg} / \mathrm{dl}$, which fell $80 \%$ to 101 $\mathrm{mg} / \mathrm{dl}$ by day 7 (Figure 1B, patient 2). Her post-transfusion hemoglobin on day 1 fell from $11.7 \mathrm{gm} / \mathrm{dl}$ to $8.2 \mathrm{gm} / \mathrm{dl}$ on day 3 , accompanied by a $32 \%$ decrease in platelet count $(365 \mathrm{~K} / \mu \mathrm{l}$ to $250 \mathrm{~K} /$ $\mu l)$. Over the first week, there was an increase in RDW (15.4 to 17.1) and MCV (84.6 fl to $87.4 \mathrm{fl}$ ). By 2 weeks, there was improvement in hemoglobin (10.2 gm/dL), RDW (15.8) and MCV (84.4 fl).

Case 2: The patient was a 50 -year-old man with history of poorly controlled hypertension who was admitted with new left-sided hemiparesis and a large intracranial hematoma involving the right temporal lobe and basal ganglia. Two weeks after admission, he developed a left upper extremity DVT. Because of his recent bleed, he was not a candidate for systemic anticoagulation and underwent an IVC filter placement. His haptoglobin decreased $57 \%$ in the first week ( 232 to $99 \mathrm{mg} / \mathrm{dl}$; Figure 1B, patient 1 ) and by $98 \%$ by day $14(5 \mathrm{mg} / \mathrm{dl})$. There was a mild decrease in platelets $(243$ to $151 \mathrm{~K} / \mu \mathrm{l})$, hemoglobin (13.2 to $11.5 \mathrm{gm} / \mathrm{dl}$ ), and increased MCV (92.1 to $94.2 \mathrm{fl}$ ): his RDW remained stable $(13.3 \pm 0.1)$. The patient was not transfused, did not receive thrombolytic therapy, had no positive cultures or evidence of infection and had normal cardiac function.

Case 3: The patient was a 48-year-old man with metastatic melanoma diagnosed one year earlier. He presented to the emergency room with headaches and was found to have a large metastasis to his left frontal lobe. He underwent surgical resection without issue. One week post-operatively, he developed a catheter-associated left upper extremity DVT. Eight days after his DVT, he developed acute dyspnea due to a large PE. An echocardiogram showed normal ventricular function and no evidence of tricuspid valve regurgitation. He was found to have multiple bilateral DVTs involving the common femoral, popliteal, posterior tibial and peroneal veins. Because of recent neurosurgery, the patient underwent IVC filter placement.

Within one week of IVC filter placement, the patient's haptoglobin fell $59 \%$ (551 to $225 \mathrm{mg} / \mathrm{dl}$; Figure 1B, patient 3). There was a $42 \%$ decrease in platelets over the first week (339 to $196 \mathrm{~K} / \mu \mathrm{l})$, accompanied by a mild decrease in hemoglobin (15.9 to $14.7 \mathrm{gm} / \mathrm{dl}$ ). He did not require transfusion. There was no prolongation of PT or PTT suggestive of a consumptive coagulopathy. The patient did have a recent catheter-associated infection with methicillin-sensitive $S$. aureus.

Several months after IVC placement, the patient was noted to have circulating nucleated $\mathrm{RBC}$, which required correction of his automated WBC counts. His hemoglobin was stable $(14 \mathrm{gm} / \mathrm{dl})$ with polychromasia, elevated MCV (102.4 fl) and increased RDW (20\%). There was no evidence of blood loss, consumptive coagulopathy or a leukoerythroblastic reaction: the corrected WBC count, WBC differential, platelet count, PT, PTT and fibrinogen remained within normal limits. His LDH was high normal (230 IU/L) with a normal bilirubin $(0.5 \mathrm{gm} / \mathrm{dl})$ : no haptoglobin was performed. An antibody screen and DAT were negative.

\section{Discussion}

IVC filters were introduced in the late 1960s as a means to prevent $\mathrm{PE}$ in patients who cannot tolerate systemic anticoagulation due to disease, surgery and trauma or who developed recurrent DVT and/or PE despite anticoagulation [22]. With the introduction of retrievable IVC filters, the use of IVC filters has increased dramatically in the United States, especially for PE-prophylaxis [22]. Known complications and adverse events associated with IVC filters include vessel erosion and perforation (0-41\%), filter fracture (2-10\%), filter movement $(0-18 \%)$, and filter embolization $(<1 \%)[22,23]$. There is also a risk of filter occlusion (2-30\%), recurrent DVT (36\%) and recurrent PE (0.5-6\%) [22]. The potential risk for low grade hemolysis and low haptoglobin levels with IVC filters is unknown and is not required by the United States Food and Drug Administration [24].

Although there are no studies on the impact of IVC filters on haptoglobin levels, older studies have shown that IVC filters can adversely affect blood rheology [25]. In the absence of trapped clots, most commercial IVC filters induce only minor disturbances in laminar flow. In the presence of trapped clots, however, all IVC filters show a marked increase in the fluid pressure gradient across the filter and turbulent blood flow downstream [25]. Turbulent, non-laminar blood flow can generate significant shear forces, resulting in red cell damage, loss of deformability and increased hemolysis [26]. It has been shown that a $1 \%$ increase in daily red cell turnover is sufficient to deplete circulating haptoglobin levels $[1,2,5]$.

In this small pilot study, we hypothesized that IVC filters may cause low grade hemolysis in some patients, leading to decreased haptoglobin levels. We therefore followed serial haptoglobins in 18 patients who underwent IVC filter placement at our institution. In most patients (group 1), haptoglobin increased post-procedure, consistent with an acute phase reaction. Studies in surgery patients have shown a steady two-fold increase in haptoglobin levels for several days post-operatively [14]. Despite the latter, studies have shown that low level hemolysis in the setting of inflammation still leads to haptoglobin depletion [5], suggesting an absence of hemolysis in our group 1 cohort.

Conversely, a small number of patients exhibited a decrease in haptoglobin following IVC filter placement. In these patients, haptoglobin levels were $<50 \%$ of pre-procedure levels by day 7 . One patient had complete depletion of haptoglobin within 2 weeks. The decline in haptoglobin was accompanied by decreases in hemoglobin and indirect evidence of reticulocytosis based on red cell indices (MCV, RDW). In one patient, nucleated RBC were noted several months after IVC filter placement. Altogether, these results suggest that 
Page 7 of 8

low grade hemolysis may occur in some patients following IVC filter placement.

Although low haptoglobin in one patient (case 1) may be attributed to a combination of thrombolytic therapy and transient pulmonary hypertension [12,13], there is not a clear explanation for the other two patients. One patient (case 2) presented with a large intracranial hemorrhage; however, three other patients with intracranial hemorrhages had normal haptoglobin levels. Furthermore, studies have shown that haptoglobin levels are not affected by internal bleeding or clot resorption, likely due to locally synthesized haptoglobin which facilitates the adsorption of hemoglobin from sites of hemorrhage $[4,5]$.

Transfusion also cannot account for the different haptoglobin responses between group 1 and 2 . In our study, 1/3 patients in group 2 and 3/6 patients in group 1 received at least one transfusion (Table 2). All patients had a negative antibody screen pre-transfusion and no transfused patient developed new RBC alloantibodies, thereby excluding haptoglobin losses due to a delayed hemolytic transfusion reaction. Furthermore, haptoglobin is not significantly affected by routine $\mathrm{RBC}$ transfusion despite recent concerns regarding $\mathrm{RBC}$ hemolysis with prolonged RBC storage [28]. Gupta and colleagues performed serial haptoglobin measurements in 21 patients who required RBC transfusion for bleeding (90\%) or acute illness [28]. Although there was a transient mild decrease $(<10 \%)$ in haptoglobin immediately post-transfusion, haptoglobin levels returned to pretransfusion levels within 24 hours. In a different study, Hod et al. showed no change in haptoglobin following transfusion of RBC that were 40-42 days of age [29]. In our study, no patient received more than 4 units and all RBC were less than 20 days of age.

Interestingly, all three patients with decreased haptoglobin levels also showed a transient fall in platelet count. This was not observed in the vast majority of patients in group 1 , who actually had an increase in platelets. A fall in platelet count could suggest trapped clot and/or thrombus formation at the IVC filter. Using indium-labeled platelets, Yagi et al. were able to demonstrate the accumulation of platelets at the site of IVC filter [30]. In another case, Nitta and colleagues documented a $25 \%$ decrease in platelets within 24 hours of IVC placement, which continued to fall over a period of 3 weeks [31]. Although the authors attributed the finding to heparin-induced thrombocytopenia, the patient's platelet count remained low for 2 weeks after discontinuing heparin and did not rebound until after the filter was removed. Studies with extracorporeal devices have shown a correlation between thrombosis and hemolysis [10].

In summary, we observed a temporal decrease in haptoglobin levels within 1 week of IVC filter placement in $3 / 18$ of patients. This was in contrast to most patients, who had a $50-100 \%$ increase in haptoglobin following filter placement. The fall in haptoglobin in some patients suggests low grade, subclinical hemolysis, possibly due to thrombus and/or trapped clot at the filter and attendant turbulent flow. It would be of interest for future studies to study IVC filters against other markers and sequlae of hemolysis including erythrocyte creatine, free hemoglobin, non-transferrin bound iron, nitric oxide and microparticle generation $[2,11,32]$.

Our study has several limitations including a small patient cohort, a short window period of observation, patient attrition and the absence of ancillary hemolysis laboratories such as LDH. LDH also has several limitations due to preanalytical variables, widespread tissue expression and poor specificity with regards to hemolysis $[5,11]$. Despite these limitations, this is the first study to prospectively examine the impact of IVC filters on haptoglobin levels. Our preliminary data suggests that IVC filters are a potential non-immune cause of low haptoglobin that should be considered when evaluating patients with anemia and low haptoglobin.

\section{References}

1. Shih AWY, McFarlane A, Verhovsek M (2014) Haptoglobin testing in hemolysis: Measurement and interpretation. Am J Hematol 89: 443-447.

2. Schaer DJ, Buehler PW, Alayash AI, Belcher JD, Vercellotti GM (2013) Hemolysis and free hemoglobin revisited: Exploring hemoglobin and hemin scavengers as a novel class of therapeutic proteins. Blood 121: 1276-1284.

3. Smith A, McCulloh RJ (2015) Hemopexin and haptoglobin: Allies against heme toxicity from hemoglobin not contenders. Front Physiol 6: 187.

4. Zhao X, Song S, Sun G, Strong R, Zhang J (2009) Neuroprotective role of haptoglobin after intracerebral hemorrhage. J Neurosc 29: 15819-15827.

5. Kormoczi GF, Saemann MD, Buchta C, Radosavlijevic MP, Mayr WR (2006) Influence of clinical factors on the haemolysis marker haptoglobin. Eur J Clin Invest 36: 202-209.

6. Mecozzi G, Milano AD, Carlo MD, Sorrentino F, Pratali S (2002) Intravascular hemolysis in patients with new-generation prosthetic heart valves: A prospective study. J Thoracic Cardio Surg 123: 550-556.

7. Mair DC, Eastlund T, Rosen G, Covin R, Harmon JV (2005) Hemolysis during percutaneous mechanical thrombectomy can mimic a hemolytic transfusion reaction. Transfusion 45: 1291-1294.

8. Nazarian GK, Qian Z, Coleman CC, Rengel G, Castaneda-Zuniga WR (1994) Hemolytic effect of the Amplatz thrombectomy device. J Vasc Interv Radiol 5: 155-160.

9. Vercaemst L (2008) Hemolysis in cardiac surgery patients undergoing cardiopulmonary bypass: A review in search of a treatment algorithm J Extra Corp Tech 40: 257-267.

10. Tchantchaleishvili V, Sagebin F, Ross RE, Hallinan W, Schwarz KQ, et al. (2014) Evaluation and treatment of pump thrombosis and hemolysis. Ann Cardiothorac Surg 3: 490-495.

11. Okumiya T, Nishi MI, Doi T, Kamoika M, Takeuchi H (2004) Evaluation of intravascular hemolysis with erythrocyte creatine in patiens with cardiac valve prostheses. Chest 125: 2115-2120.

12. Kline JA, Marchick MR, Hogg MM (2009) Reduction in plasma haptoglobin in humans with acute pulmonary embolism causing tricuspid regurgitation. J Thromb Haemost 7: 1597-1599.

13. Insenser M, Nieto RM, Garcia MAM, Duran EF, Santiuste C (2014) Identification of reduced circulating haptoglobin concentration as a biomarker of the severity of pulmonary embolism: a nontargeted proteomic study,

14. Fischer CL, Gill C, Forrester MG, Nakamura R (1976) Quantitation of "acute-phase proteins" postoperatively. Am J Clin Path 66: 840-846.

15. Pan JP, Cheng TM, Shih CC, Chiang SC, Chou SC (2011) Haptoglobin phenotypes and plasma haptoglobin levels in patients with abdominal aortic aneurysm. J Vasc Surg 53: 1189-1194.

16. Belij S, Miljikovic D, Popov A, Subota V, Timotijevic G (2012) Effects of subacute oral warfarin administration on peripheral blood granulocytes in rats. Food Chem Toxicol 50: 1499-1507.

17. Roberts GT, Badawi BDEl (1985) Red blood cell distribution width index in some hematological disease. Am J Clin Path 83: 222-226.

18. Sayed HLE, Tawfik ZM (1994) Red cell profile in normal and sickle cell disease in children. J Egypt Soc Parasitol 24: 147-154.

19. Bowen DT, Culligan D, Beguin Y, Kendall R, Willis N (1994) Estimation of effective and total erythropoiesis in myelodysplasia using serum transferring and erythropoietin concentrations, with automated reticulocyte parameters. Leukemia 8: 151-155.

20. Chen L, Wu Z (1998) Study on the clinical diagnostic values of MCV and RDW to hemolytic anemia. Bulletin Hunan Med University 3: 203-205. 
Citation: Cooling L, Giacherio D, Elkins M (2017) Inferior Vena Cava Filters as a Potential Non-immune Cause of Low Serum Haptoglobin. J Blood Disord Transfus 8: 382. doi:10.4172/2155-9864.1000382

Page 8 of 8

21. Reidl J, Posch F, Konigsbrugge O, Lotsch F, Reitter EM (2014) Red cell distribution width and other red blood cell parameters in patients with cancer: Association with risk of venous thromboembolism and mortality.

22. Wang SL, Lloyd AL (2013) Clinical review: Inferior vena cava filters in the age of patient-centered outcomes. Ann Med 45: 474-481.

23. Sella DM, Oldenburg WA (2013) Complications of inferior vena cava filters. Sem Vasc Surg 26: 23-28.

24. (1999) Food and Drug Administration. Guidance for cardiovascular intravascular filter $510(\mathrm{~K})$ submissions.

25. Katsamouris AA, Waltman AC, Delichatsios MA, Athanasoulis CA (1988) Inferior vena cava filters: in vitro comparison of clot trapping and flow dynamics. Radiology 166: 361-366.

26. Kameneva MV, Burgreen GW, Kono K, Repko B, Antaki JF, et al. (2004) Effects of turbulent stresses upon mechanical hemolysis: experimental and computational analysis. ASAIO J 50: 418-423.

27. Gladwin MT, Shapiro DBK (2009) Storage lesion in banked blood due to hemolysis-dependent disruption of the nitric oxide homeostasis. Curr Opin Hematol 16: 515-523.
28. Gupta S, Ahern K, Nakhl F, Forte F (2011) Clinical usefulness of haptoglobin levels to evaluate hemolysis in recently transfused patients. Adv Hematol.

29. Hod EA, Brittenham GM, Billote GB (2011) Transfusion of human volunteers with older, stored red blood cells produces extravascular hemolysis and circulating non-transferrin-bound iron. Blood 188: 6675-6682.

30. Yagi A, Oriuchi N, Sato N, Aoki J, Higuchi T, et al. (2004) Pulmonary thromboembolism-evaluating the indication and effect of a vena caval filter with indium-111-platelet scintigraphy. Circ J 68: 599-601.

31. Nitta N, Shitar S, Nozaki K (2011) Heparin-induced thrombocytopenia in a gliobastoma multiforme patient with inferior vena cava filter placement for deep venous thrombosis. Neurol Med Chir (Tokyo) 51: 445-448.

32. Westerman M, Porter JB (2016) Red blood cell-derived microparticles: An overview. Blood Cells, Molecules and Diseases 59: 134-139. 